IFT-UAM-97-5

hep-th/9712136

\title{
NEWTONIAN M(ATRIX) COSMOLOGY
}

\author{
Enrique Álvarez and Patrick Meessen \\ Instituto de Física Teórica, CXVI \\ and \\ Departamento de Física Teórica,CXI \\ Universidad Autónoma, 28049 Madrid, Spain \\ enrial,meessen@daniel.ft.uam.es
}

\begin{abstract}
A Newtonian matrix cosmology, corresponding to the Banks, Fischler, Shenker and Susskind model of eleven-dimensional M-theory in the infinite momentum frame as a supersymmetric (0+1) $\mathrm{M}$ (atrix) model is constructed. Interesting new results are obtained, such as the existence of (much sought for in the past) static solutions. The possible interpretation of the off-diagonal entries as a background geometry is also briefly discussed.
\end{abstract}


One of the most interesting recent results in string theory $1 \|$ is that the strong coupling limit of type IIA strings is some unknown eleven dimensional theory, whose low energy limit is $\mathrm{N}=1$ supergravity in 11 dimensions.

Although this result, combined with the general framework of string dualities, allows renewed hopes on the dream of unification, the fact that the eleven dimensional M-Theory does not have any known moduli associated to a coupling constant makes further progress difficult.

One of the few attempts to describe in a concrete way M- Theory is the M(atrix) model of Banks, Fischler, Shenker and Susskind [2, 3]. The starting point is the seminal idea of 5 on the general description of a system of a given number, $\mathrm{N}$, of $\mathrm{D} 0$-branes by means of the dimensional reduction of super-Yang-Mills in $(1+9)$ dimensions to $(1+0)$ dimensions, i.e. a supersymmetric quantum mechanical model with bosonic degrees of freedom given by nine $\mathrm{N}$ by $\mathrm{N}$ (hermitian) matrices in the Lie algebra of $\mathrm{U}(\mathrm{N}), X^{i}$. To be specific, the bosonic part of the action is:

$$
S=\int d t \operatorname{tr}\left(\frac{1}{2 R_{c}}\left(D_{0} X^{i}\right)^{2}+\frac{R_{c}}{4 l_{11}^{6}}\left(\left[X^{i}, X^{j}\right]\right)^{2}\right.
$$

where $R_{c}$ is the proper size of the spacelike circle (becoming lightlike when $\epsilon \downarrow 0$; this is a technical point discussed by Bigatti and Susskind in [3]) and $l_{11}$ is the scale set by the eleven-dimensional Newton's constant, $G_{11} \equiv l_{11}^{9}$.

The bold hypothesis of BFSS is that precisely this lagrangian (when supersymmetrized) describes the 11-dimensional dynamics of M-theory, albeit in a frame boosted in the $x^{11}$ direction, the infinite momentum frame, IMF. (widely used to describe some aspects of the physics of partons in the past [7]). It has been proven that it reproduces low energy supergraviton scattering [8], and also membranes, following earlier suggestions in [6]. In order to be able to consider arbitrary sets of particles, it seems necessary to take the limit $N \rightarrow \infty$.

It is not clear what precisely the physical meaning of the fact that $\mathrm{D}$ 0-branes are described by "coordinates-matrices" is. On the one hand, it is clear that the eigenvalues of the matrices (wich will be denoted throughout by $y_{a}^{i}$, where $a=1, \ldots, N$ ) label the ordinary positions, whereas the offdiagonal terms (denoted generically by $z_{a b}^{i}$ ) are related to the interactions between the particles themselves . It could be that Nature is described

\footnotetext{
${ }^{1}$ This position seems to be at variance with the one expressed in [2], in which the relative distance between two separate "clusters" in a block-diagonal matrix, with blocks
} 
at some fundamental level by non-conmutative geometry, and that nonseparability is built in the theory at a fundamental level.

At any rate, it seems clear that the model captures at least some relevant aspects of interesting eleven dimensional physics.

\section{2}

In the IMF relativistic invariance is hidden, and only galilean one is manifest. Energies are given up to a constant by $H=\frac{k_{\perp}^{2}}{2 \eta}$, where the "Newtonian" mass is related to the eleventh component of the momentum in the IMF, $k_{11} \equiv$ $\frac{\eta}{\sqrt{2}} e^{\omega}$, where $\omega$ is the hyperbolic rotation angle determining the boost $(\omega \rightarrow$ $\infty)$. The energy in the unboosted frame is clearly given by $p_{0} \equiv \frac{1}{\sqrt{2}}\left(\eta+\frac{k_{\perp}^{2}}{2 \eta}\right)$, and the unboosted eleventh component of the momentum is $p_{11} \equiv \frac{1}{\sqrt{2}}(\eta-$ $\left.\frac{k_{\perp}^{2}}{2 \eta}\right)$. Another point of view, pioneered by Susskind, and briefly alluded to in the prevoius section, starts from discrete light cone quantization, in which the light-like coordinate $x^{-}$is compactified to a circle of circumference $2 \pi R$. This means that the spectrum of $p_{-}(\equiv \eta)$ is discretized: $p_{-}=N / R$, and we assume that $N$ (to be identified later on with the number of partons) is a non-negative integer. Eventually the limit $N \rightarrow \infty$ is necessary.

This means that if we want to study cosmology in this frame (the purpose of the present paper), it would be some form of Newtonian cosmology. It could be thought premature to speculate in this direction, but in our opinion it is always useful to be able to imagine a cosmological scenario compatible with the best candidate to date for a fundamental theory $\mathrm{L}^{2}$.

Newtonian cosmology is a beautiful theory. It is almost a deductive one; starting from the cosmological principle (postulating that the universe presents the same aspect from every point except for local irregularities) and assuming further that there is a universal force (the cosmological constant) proportional to the distance, all results of the theory flow smoothly [11].

As has been stressed by Bondi, the main subject is the study of the motion of the substratum, which for our purposes will be idealized as the streaming of a uniform fluid. The concrete implementation of the cosmological principle will stem from the assumption that to all comoving observers

$Y_{a}\left(N_{a} \times N_{a}\right)$ and $Y_{b}\left(N_{b} \times N_{b}\right)$, with $N=N_{a}+N_{b}$ is $R_{a, b} \equiv\left|\frac{\operatorname{tr} Y_{a}}{N_{a}}-\frac{\operatorname{tr} Y_{b}}{N_{b}}\right|$, but it is nevertheless the simplest way of getting gauge invariant expressions for the coordinates themselves, a very desirable property from our point of view.

${ }^{2}$ Some previous attempts in the broad framework of Kaluza-Klein cosmology are to be found in $[12]$. 
the model presents the same appearance (at a given Newtonian time).

Although this is the standard formulation of the cosmological principle, given the fact that the object of interest in the present work is a Newtonian theory with extra dimensions, some modifications can be thought of. It could be applied to the ordinary three spatial dimensions only, for example, which would constitute a sort of "broken symmetry" phase. From this point of view, the present work is restricted to the symmetric phase. (Non-symmetric phases would be related to the study of cosmological (timedependent) solutions of ordinary supersymmetric Yang-Mills field theory (in $\mathrm{n}+1$ dimensions), at least for toroidal $\mathbb{T}^{n}$ compactifications, according to the standard procedure [9]). Another window this sort of considerations would open up is the cosmological study of the effects of T-duality [14 in this context.

The cosmological principle implies striking consequences on the allowed velocity field for the substratum. Let us consider two fundamental (that is, comoving with the substratum) observers, $O$ and $O^{\prime}$, measuring the velocity of a given point $P$. Observer $O$ will report $V^{i}\left(X^{j}\right)$, and observer $O^{\prime}$ will report $V^{\prime i}\left(X^{\prime j}\right)$. Uniqueness of the velocity at $P$ (plus the Newtonian law of addition of velocities) then necessarily implies that $V^{\prime i}\left(X^{\prime j}\right)=$ $V^{i}\left(X^{j}\right)-V^{i}\left(X^{j}-X^{\prime j}\right)$ Now, the cosmological principle imposes $V^{\prime}(x) \equiv$ $V(x)$, which means that the velocity field must be linear in the coordinate labels: $V^{i}\left(X^{j}\right)=A X^{i} B$, where $A$ and $B$ are $N \times N$, possibly, time-dependent matrices.

We are assuming here that the substratum, is constituted somehow of D-0 branes, in a regime in which a matrix description of them is compulsory. A fascinating question, which has been left out for future work, is the construction of a dual, complementary description in terms of membranes.

In the standard scalar case, isotropy further requires that there is a single function of time, $f(t)$ such that $\vec{v}=f(t) \vec{r}$. If we want this property to be preserved on the eigenvalues of the coordinate matrix $X$, and further demand hermiticity, we are left with

$$
V^{i}\left(X^{j}\right) \equiv f(t) S X^{i} S^{+}
$$

where $S$ is a unitary matrix. In the scalar case, this equation can be further integrated to $\vec{r}=R(t) \vec{r}_{0}$, with $f \equiv \dot{R} / R$. A similar Ansatz in the present situation would be

$$
X^{i} \equiv R(t) U X_{0}^{i} U^{+}
$$


again, with a unitary $U$, yielding

$$
\dot{X}^{i}=\frac{\dot{R}}{R} X^{i}+R U\left[U^{-1} \dot{U}, X_{0}^{i}\right] U^{-1}
$$

Assuming that the stress-energy from all matter of the Universe is dynamically negligibles], the only remaining force is the cosmological constant $F^{i}=\lambda X^{i}$, (introduced in the newtonian context by Neumann and Seeliger in 1896 [1]) which means that the dynamical Newtonian equations following from the BFSS action principle (after a trivial rescaling) are

$$
\ddot{X}^{i}+\sum_{j}\left[X^{j},\left[X^{j}, X^{i}\right]\right]=\lambda X^{i}
$$

The preceding equations convey an interesting generalization of the standard vacuum equations of scalar Newtonian cosmology. The eigenvalues of the coordinate-matrices obey exactly the standard equations as in [11]. (Nevertheless, if the unitary matrix $U$ is non-trivial the labels of the eigenvalues suffer a time-dependent permutation). The most conservative interpretation of the other entries of the matrices is that they are related to the interactions. More on this later.

Although it is not the purpose of the present work to be exhaustive about it, there are very interesting new solutions. One of the simplest stems from the assumption that the unitary matrix $U=1$, leading to

$$
\ddot{R} X_{0}^{i}+R^{3} \sum_{j}\left[X_{0}^{j},\left[X_{0}^{j}, X_{0}^{i}\right]\right]=\lambda R X_{0}^{i}
$$

The trace of the above is

$$
(\ddot{R}-\lambda R) \operatorname{tr} X_{0}^{i}=0
$$

There are then two possibilities: either $\operatorname{tr} X_{0}^{i} \neq 0$, in which case the standard scalar result is recovered: $R=R_{0} \cosh \left(\sqrt{\lambda}\left(t-t_{0}\right)\right.$ ) (if $\lambda>0$ ), or $R=$ $R_{0} \cos \left(\sqrt{-\lambda}\left(t-t_{0}\right)\right.$ ) (if $\lambda<0$ ); or else $\operatorname{tr} X_{0}^{i}=0$, which means that $X_{0}^{i}$ can be expanded in generators of $\mathrm{su}(\mathrm{n}): X_{0}^{i} \equiv \sum_{a} u_{i a} T_{a}$. To be specific, assuming the simplest case $N=2$, and $u_{i a}=\theta(3-i) u_{i a}$, with furthermore $\sum_{c} u_{i c} u_{j c}=\frac{1}{2} \delta_{i j}$ (equivalent to choosing a set of 3 orthonormal ordinary three-vectors ), then the $u_{i a}$ are subject to no further restriction, whereas $\mathrm{R}$ obeys:

$$
\ddot{R}-\lambda R+R^{3}=0
$$

\footnotetext{
${ }^{3}$ This assumption could easily be bypassed
} 
whose solutions are, generically, given in terms of elliptic funcions.

There is a fascinating particular solution, though, in the case $\lambda>0$, and it is a static one, $R=\sqrt{\lambda}$. This is very curiousf, because (scalar) Newtonian Cosmology was abandoned essentially since the first attempts in the nineteenth century because it does not allow static solutions, and this was exactly everybody's prejudice until Milne and MacCrea revitalized the subject (in 1934) drawing on insights from relativistic cosmology as well as on Hubble's data on the expansion of the Universe.

Apart from the static solution, $R=$ constant, we can multiply the above equation with $\dot{R}$ and find

$$
\dot{R}^{2}-\lambda R^{2}+\frac{1}{2} R^{4}=\mathcal{C},
$$

where $\mathcal{C}$ is some constant. One one can follow 11] in his analysis of solutions, but for the sake of briefness let's give the explicit expression for the case $\lambda=0$ : Integration imposes that $\mathcal{C} \geq 0$, and we find that the solution is given by [15]

$$
R(t)=a \mathbf{s d}\left(\sqrt{2} a\left(t-t_{0}\right) \mid \frac{1}{2}\right)
$$

where $a^{4}=\mathcal{C}$.

The above solution can be easily generalized to arbitrary $N$. Note that the solution is based on the asumption that we have matrices $X_{0}$ such that

$$
\sum_{j}\left[X_{0}^{j},\left[X_{0}^{j}, X_{0}^{i}\right]\right] \equiv X_{0}^{i}
$$

If we now decompose the $X$ 's on a base of $s u(N)$, using the Lie-data $\left\{T_{a}, f_{a b}^{c}\right\}$, we see that the above equation reads

$$
X^{i a} \sum_{j} X^{j b} X^{j c} f_{a b}^{d} f_{e d c}=X_{e}^{i}
$$

where we have used the Killing metric on $s u(N), g_{a b}$ say, to lower the index. It is then easy to see that iff we take the $X^{j b}$ such that, reverting to vector notation,

$$
\sum_{j} \vec{X}^{j}\left(\vec{X}^{j}\right)^{T}=g,
$$

\footnotetext{
${ }^{4}$ Although perhaps not so surprising, given the fact that our building blocks for the substratum are D-0 branes, which are well known to be BPS states, and, as such, able to survive in static configurations of equilibrium
} 
we automatically satisfy the above equation, due to the definition of the Killing metric. Note that we are using Hermitean generators, so that the Killing metric is positive definit.

A completely different solution is obtained for one-dimensional motion (still with $\mathrm{N}=2$, for simplicity). A rotation can always be made so that $X_{0}=\sigma_{3}$. Making the general ansatz $U=u^{0}+i \vec{u} \vec{\sigma}$, it follows easily that

$$
X=R(t)\left(\begin{array}{cc}
1-2 \sin ^{2} \theta(t) & -2 \sin \theta(t) \cos \theta(t) e^{i \phi(t)} \\
-2 \sin \theta(t) \cos \theta(t) e^{-i \phi(t)} & 2 \sin ^{2} \theta(t)-1
\end{array}\right)
$$

The simplest case would be $\theta=\pi / 4$, giving

$$
\begin{gathered}
\ddot{R}-R \dot{\phi}^{2}-\lambda R=0 \\
R \ddot{\phi}+2 \dot{R} \dot{\phi}=0
\end{gathered}
$$

Again, a curious thing about these equations is that they allow static solutions when $\lambda<0 ; \phi=\sqrt{-\lambda} t+\phi_{0}$ and $R=R_{0}$ arbitrary.

Given the fact that the matrix $U$ is not trivial anymore, there is now a time-dependent permutation of the labels of the two fundamental particles in our oversimplified model.

\section{3}

The problem of the physical interpretation of the off-diagonal terms must now be tackled. The sort of solutions presented here (at least when $\lambda=0$ ) correspond to particles as free as they can be in the model. The most conservative approach would be to identify a spacetime metric such that their geodesics coincide with the trajectories of the eigenvalues in $\mathrm{M}$ (atrix) Cosmology. There are problems, however. The hamiltonian of the M(atrix) model yields an expression for the energy, which, when the effects on the eigenvalues $y_{a}^{i}$ are separated from the rest $\left(z_{a b}^{i}\right)$ is, in a somewhat symbolic notation,

$$
E=\frac{1}{2} \sum_{a=1}^{N}\left(\dot{y}_{a}^{i}\right)^{2}+g_{a b}^{i j} \dot{z}_{a}^{i} \dot{z}_{b}^{j}+V\left(y_{a}^{i}, z_{b c}^{j}\right)
$$

where the $g_{a b}^{i j}$ are quadratic functions of the $y_{a}^{i}$. This means that the classical equations of motion constitute a coupled system of $N^{2}$ differential equations of the type:

$$
\ddot{y}_{a}^{i}=F_{a}^{i}\left(y_{b}^{j}, \dot{z}_{c d}^{k}, z_{e f}^{l}\right)
$$


where the functions $z_{a b}^{i}(t)$ obey:

$$
\ddot{z}_{a b}^{i}=G_{a b}^{i}\left(y_{a}^{i}, \dot{y}_{b}^{j}, y_{c}^{k}, z_{d e}^{l}\right)
$$

In the limit $N \rightarrow \infty$ we can assume the we have an $\infty^{9}$ of fundamental observers, $a \equiv a^{i} \in \mathbb{R}^{9}$, such that for each point there is a unique trajectory passing through it. There are many curved spaces such that the trajectories $y_{a}^{i}=y_{a}^{i}(t)$ are geodesics of it; it suffices to take the congruence of fundamental observers as timelike lines; parametrized by $x^{i}=a^{i}$, (so that the change of coordinates is defined by $x^{i}=a^{i}\left(y^{k}, t\right)$ ) and a metric, for example such as

$$
d s^{2}=d t^{2}-R(t)^{2} \delta_{i j} d x^{i} d x^{j}
$$

(which reduces to $d s^{2}=d t^{2}$ on the trajectories by using $\frac{\partial a^{i}}{\partial y^{k}} \dot{y}^{k}+\dot{a}^{j}=0$ ).

In the initial coordinates it reads:

$$
d s^{2}=\left(1-R^{2} \dot{a}^{2}\right) d t^{2}-R^{2} \delta_{i j} \frac{\partial a^{i}}{\partial y^{k}} \frac{\partial a^{j}}{\partial y^{l}} d y^{k} d y^{l}-2 R^{2} \delta_{i j} \frac{\partial a^{i}}{\partial y^{k}} \frac{\partial a^{j}}{\partial t} d y^{k} d t
$$

There is a certain latitude as to how to choose the function $R(t)$. The simplest way would be to demand the we recover newtonian cosmology on the constant time hypersurfaces (that is, on the 9-space orthogonal to the trajectories of our fundamental observers). This leads to its identification with the same function $R(t)$ of preceding paragraphs.

Neither should constitute a big surprise the fact that the above metric is not flat in general. D-0-branes only feel the metric and the RR-vector, so that the background constructed as above need, a priori, not satisfy the IIA-Sugra equations. A legitimate question is whether, building on the data obtained from M(atrix), we can find a IIA-Sugra solution a posteriori.

Another point is that this technique gives naturally a 10-dimensional background. It could be thought more natural to interpret it as an 11dimensional one, but it does not appear easy in our framework (other than definining 11-dimensional decompactifications of the 10-dimensional manifold $\mathrm{M}$ ).

\section{4}

Quantum effects in the IMF are usually simpler to compute, because vacuum effects are absent. This is due to the fact that as the boost parameter $\omega \rightarrow \infty$ those processes corresponding to diagrams which have internal lines carrying negative $\eta$ will go to zero, and only those with positive $\eta$ survive. Quantum 
cosmology would then be particularly transparent in this frame, in which the physical interpretation of the wave function of the universe could follow the guidelines of hadronic wavefunction in parton physics. We hope to be able to return to this topic in the future.

It is perhaps worth stressing, to conclude, that the set of ideas here introduced with a motivation based on M-Theory, could also be contemplated as a natural generalization of Newtonian Cosmology per se. The basic equations could easily have been derived just after the introduction of the matrix concept by Sylvester around 1850 .

\section{Acknowledgements}

We have benefited from many discussions with A. Casas, C. Gómez and T. Ortín. This work has been supported by EU TMR contracts ERBFMRXCT960012 and ERBFMBICT960616, and CICYT grants AEN/96/1655 and AEN/96/1664.

\section{References}

[1] E. Witten: "String Theory Dynamics in Various Dimensions", Nucl. Phys. B443(1995), 85, hep-th/9503124.

[2] T. Banks, W. Fischler, S. H. Shenker and L. Susskind: "M-Theory as a Matrix Model: a conjecture" hep-th/9610043; Phys. Rev. D55(1997), 5112, hep-th/9610043.

[3] A. Bilal: "M(atrix) theory: a pedagogical introduction", hepth/9710136; D. Bigatti, L. Susskind: "Review of Matrix Theory", hepth/9712072.

[4] J. Polchinski: "TASI lectures on D-branes", hep-th/9611050.

[5] E. Witten: "Bound states of strings and p-branes", Nucl. Phys. B460(1996), 335, hep-th/9510135.

[6] B. de Wit, M. Lüscher, H. Nicolai: Nucl. Phys. B305(1988), 545.

[7] J. Kogut, L. Susskind: "The parton picture of elementary particles", Phys. Rep. 8C(1973), 75. 
[8] C. Bachas: "D-brane dynamics", Phys. Lett B374(1996), 37, hepth/9511043; K. Becker, M. Becker: "A two-loop test of M(atrix) theory", hep-th/9705091.

[9] W. Taylor: "D-brane field theory on compact spaces", Phys. Lett B394(1997), 283, hep-th/9611042.

[10] A. Connes: "Non-Commutative Geometry", Academic Press (1994).

[11] H. Bondi: "Cosmology", Cambridge University Press, Cambridge (1952).

[12] P.G.O. Freund, M. Rubin: Phys. Lett. 97B(1980), 233; P.G.O.Freund: Nucl. Phys. B209(1982), 146; E. Alvarez, M.B. Gavela: Phys. Rev. Lett. 51(1983), 931; E. Alvarez: "Cosmological Solutions of N=1 Supergravity in 11 Dimensions", Phys. Rev. D30(1984), 1394; N. Kaloper, I.I. Kogan, K.A. Olive: "Cos(M)ological Solutions to M-and String Theory", hepth/9711027.

[13] L. Susskind: "Another Conjecture about M(atrix) Theory", hepth/9704080.

[14] A. Giveon, M. Porrati, E. Rabinovici: "Target Space Duality in String Theory", hep-th/9401139; E. Alvarez, L. Alvarez-Gaumé, Y. Lozano: "An Introduction to T-Duality in String Theory", Nucl. Phys. (Proc. Pub.) 41(1995), 1.

[15] M. Abramowitz, I.A. Stegun: "Pocketbook of Mathematical Functions", Verlag Harri Deutsch (1984). 